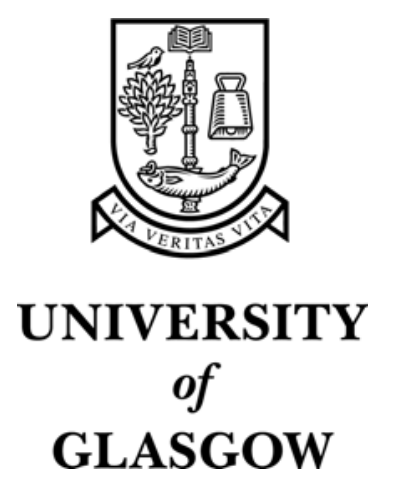

Kolch, W. and Calder, M. and Gilbert, d. (2005) When kinases meet mathematics: the systems biology of MAPK signalling. FEBS Letters 579(8):pp. 1891-1895.

http://eprints.gla.ac.uk/2871/ 


\section{When kinases meet mathematics: the systems biology of MAPK signalling}

Walter Kolch ${ }^{1,2}$, Muffy Calder ${ }^{3}$ and David Gilbert ${ }^{3}$

${ }^{1}$ Sir Henry Wellcome Functional Genomics Facility, Institute of Biomedical and Life Sciences, University of Glasgow, Glasgow G12 8QQ, UK

${ }^{2}$ Beatson Institute for Cancer Research, Garscube Estate, Glasgow G61 1BD, UK

${ }^{3}$ Department of Computing Science, University of Glasgow, Glasgow G12 8QQ, UK

Corresponding author

Walter Kolch

Beatson Institute for Cancer Research

Garscube Estate

Glasgow G61 1BD

UK

Tel: ++44 - 141 - 3303983

Fax: $++44-141-9426521$

Email: wkolch@beatson.gla.ac.uk 


\begin{abstract}
The MAPK/ERK pathway regulates fundamental cellular function such as cell proliferation, survival, differentiation and motility, raising the question how these diverse functions are specified and coordinated. They are encoded through the activation kinetics of the pathway, a multitude of feedback loops, scaffold proteins, subcellular compartmentalisation, and crosstalk with other pathways. These regulatory motifs alone or in combination can generate a multitude of complex behaviour. Systems biology tries to decode this complexity through mathematical modelling and prediction in order to gain a deeper insight into the inner works of signalling networks.
\end{abstract}

Key words: $M A P K / E R K$, signalling networks, systems biology, computational modelling 


\section{The MAPK pathway}

Originally, the name Mitogen Activated Protein Kinase, MAPK, referred to a kinase that was rapidly stimulated by insulin and other growth factors. When cloned this kinase was dubbed Extracellular signal Regulated Kinase, ERK, and the name MAPK evolved into the family name for a still growing number of related kinases that respond to stress and other stimuli $[1,2]$. Here we use the names MAPK and ERK synonymous. The MAPK pathway is one of the most intensely studied signalling pathways. It was one of the first connections between extracellular cues and changes in gene expression that had been mapped in molecular detail, and it turned out to be involved in the control of a bewildering number of cellular processes including fundamental functions such as cell proliferation, survival, motility, and differentiation. The basic topology of the pathway and its biochemistry are simple [3] (Fig.1). The activation of surface receptors leads to the activation of the membrane resident GTPase Ras which recruits a Raf kinase from the cytosol to the cell membrane. Here, Raf is activated through a still not completely known process that involves interaction with adaptor proteins and changes in phosphorylation. Although all three Raf family members, Raf-1, B-raf and ARaf, are activated by Ras, the exact mode of activation, exhibits some salient differences in particular pertaining to requirements for co-factors and phosphorylation [4]. However, all Raf family isozymes can phosphorylate and activate another kinase, MEK, which in turn phosphorylates and activates ERK. While Raf and MEK have a very restricted set of substrates, ERK features more than 70 substrates including nuclear transcription factors.

\section{Why do we need a systems biology approach to understand signalling?}

The apparent biochemical simplicity of the MAPK pathway is in stark contrast to its pleiotropic cellular functions posing a burning question. How does this pathway specify different biological responses? This riddle applies to all signalling pathways. The amplitude 
and duration of the signal flux through a pathway may determine the biological outcome. The classical example is the PC12 cells where the sustained activation of ERK triggers neuronal differentiation, while transient ERK activity is required for proliferation [5]. However, it is largely unknown how biological response specificity is encoded through biochemical activation kinetics. In addition, pathways are traditionally drawn as separated linear entities. However, this rather reflects the history of how they were discovered than their real functional context. It has turned out that pathways are extensively connected and embedded in networks. The economy of evolution has produced a multitude of links and crosstalk between pathways and even led to the reuse of protein components in different pathways. Thus, the specificity of biological responses is largely generated by the combinatorial integration of pathway crosstalk and the versatility of component function.

Systems biology aims to understand biological behaviour at the systems level through an abstract description in terms of mathematical and computational formalisms. In order to be useful and applicable to biological questions, models have to faithfully describe the biological system and be able to make predictions about their behaviour. Thus, while the basis of a model is the topological representation of its components and their links, it is the description in the model of the biological system's dynamic behaviour which equips the model with predictive power. This power can then be exploited by incorporating descriptions of perturbations of the biological system into the model and using computational techniques to predict possible behaviours of the system. In a model perturbations can be made at any component individually or at several components simultaneously and at any point in time. This strategy can be used to define the master switches where small changes have a big impact on system behaviour and the thresholds that delineate true signals from background noise. This information permits the design experiments that capture systems behaviour by 
measuring selected parameters, which are representative for the system, rather than the whole system. This reduces the number of biological experiments required to understand the behaviour of a system and also guides parameter choice for quantitative experimentation. Further, and maybe most stimulating mathematical modelling can reveal and explain unexpected behaviour that is encoded in the design of biological networks. Several models of the MAPK pathway are available which have emphasised different facets $[6,7,8,9,10,11,12,13,14,15]$, and we will refer to these papers as we discuss the salient findings in the field.

\section{Is more better than one: Three tiered cascades and dual phosphorylations}

MAPK/ERK is activated by a linear sequence of two other kinases, MEK and Raf. Why have two more kinases to process the output of one? Intuitively, signal amplification comes to mind. However, the signal gain is rather modest [16] and if it occurs, mainly occurs at the MEK - ERK interface [6]. This suggests that a main purpose is regulation. A multicomponent cascade has more interfaces available for regulation improving the fine tuning of signal flux through intra-cascade feedback regulation and cross talk with other pathways. For instance, the activation of Phosphoinositide-3 kinase (PI3K) triggers an activating phosphorylation of Raf-1 on S338, and in some cell types an inactivating phosphorylation on S259 [17]. S338 phosphorylation is mediated through a PI3K-Rac-PAK pathway, while S259 phosphorylation is mediated through a PI3K-Akt pathway. In addition, the PI3K-RacPAK pathway also can phosphorylate MEK on S298 to increase its ability to become activated by Raf [18]. Thus, at the level of the Raf-MEK interface alone PI3K can mediate at least three regulatory events, two of which have opposite effects. In cases like this intuition fails us in predicting the outcome. Only a mathematical model will be able to predict what exactly happens to MEK activity when PI3K is switched on. One such model has been 
developed showing that only the inhibitory phosphorylation of Raf-1 on S259 by Akt in combination with a phosphatase, PP2 that is shared between the two pathways could accurately predict the behaviour of ERK activity measured biochemically [13].

Another peculiarity is that both MEK and ERK require a double phosphorylation to become activated. This gives rise to some interesting behaviour that would not have been spotted without mathematical analysis. The salient work [19] was done in Xenopus oocytes where progesterone treatment triggers ERK activation and subsequent maturation. Xenopus oocytes are large enough to study ERK activation biochemically in single cells rather than in the usual large populations. While the population showed an apparently linearly graded activation of ERK in response to increasing levels of progesterone, individual eggs exhibited an all or nothing response. It was a gradual increase in the frequency of responding cells that gave rise to the linear response curve of the population. The underlying mechanism was traced to the non-processivity of ERK phosphorylation. When MEK encounters ERK it phosphorylates only one of the two residues required for activation before it dissociates. Thus, a suboptimal stimulus will generate a pool of single phosphorylated, but inactive ERK. Any subsequent phosphorylation of this monophosphorylated pool will result in activation. Kinetically this system behaves like a co-operative enzyme which can be described by the Hill equation. As a result ERK activation kinetics adopts a sigmoidal shape becomes switchlike. This phenomenon is called ultrasensitivity. It is ideally suited to filter out background noise. Physiologically cells are exposed to a sea of external cues, and sorting out true signals from noise is an essential task. However, it is not resolved whether MAPK ultrasensitivity exists in mammalian cells. It has been shown at the population level, but a recent study [20] at both population and single cell level has argued that in mammalian cells the pathway is 
stimulated with linear dose response kinetics. This discrepancy could potentially be explained by the differential expression of scaffolding proteins.

\section{Order from chaos: Feedback loops}

Feedback loops in biological systems have received a lot of attention from theoreticians as they can be well described mathematically, are versatile regulators that produce interesting behaviours and are used in many engineering applications. The MAPK features a series of positive and negative feedback regulations. Their description in this review is not exhaustive, but rather meant to illustrate how feedback is used to generate distinct biochemical and biological behaviour.

ERK feeds back to MEK activation at several levels. MEK needs to interact with Raf in order to become efficiently phosphorylated on the activating sites S218 and S222. This interaction is highly regulated. In response to adhesion PAK1 phosphorylates MEK on S298, which enhances its interaction with Raf. Activated ERK can phosphorylate MEK on the adjacent T292 which precludes phosphorylation of S298 and reduces the formation of RafMEK complexes and MEK activation [18]. The interaction between Raf and MEK is also regulated by RKIP, a protein that binds to Raf and MEK preventing their interaction [21]. Phosphorylation of RKIP by either PKC [22] or ERK [23] causes RKIP to dissociate and Raf-MEK interaction to proceed. ERK mediated phosphorylation of RKIP constitutes a positive feedback loop that converts the linear dose response relationship expected from a stoichiometric inhibitor into a steep sigmoidal curve [23]. In most cells part of the Raf population is bound to RKIP, which does not prevent it from becoming activated, but from access to its substrate MEK. The feedback phosphorylation of RKIP by ERK thus releases active Raf that is immediately available to phosphorylate MEK and activate the pathway with very steep kinetics. Likewise, a gradual overexpression of RKIP results in an abrupt 
inhibition as the positive feedback loop keeps the pathway functioning until a critical amount of Raf is sequestered by RKIP.

Feedback loops also can be used to sense the duration of signals. For instance, in Swiss 3T3 both EGF and PDGF induce the expression of the $c$-fos gene with similar efficiency and initial kinetics via the activation of ERK [24]. However, in EGF treated cells ERK is rapidly dephosphorylated. Due to the time required for transcription and translation the c-Fos protein expression peaks only after ERK activity has ceased and c-Fos is rapidly degraded. In contrast, PDGF sustains ERK activation and stabilises the c-Fos protein for many hours permitting the cell to enter the cell cycle and divide. Protein stabilisation is due to the phosphorylation of c-Fos by ERK and the ERK activated kinase RSK. Thus, the different duration of ERK signalling is biologically sensed as differences in c-Fos protein stability as result of at least two feedback loops and a time delay: a negative feedback that inactivates EGF stimulated ERK before the c-Fos protein is produced, and a positive feedback which allows ERK to stabilise c-Fos through phosphorylation.

\section{Feedback, decision making and biological memory}

Ultrasensitivity and positive feedback can generate switch-like systems which are only stable in on or off configurations $[25,26]$. In ultrasensitive systems this is achieved through the input parameters crossing a certain threshold value that switches the response. Positive feedback can generate bistable systems where two discrete stable states exist for a single input value. Although a positive feedback (or equivalent double negative feedback) is commonly considered necessary for generating bistability, a double non-processive phosphorylation can suffice under certain conditions to evoke bistable behaviour. Bistable systems show hysteresis meaning that the dose response curve splits into a loop indicating that the stimulus needed to activate the system is quantitatively different from the stimulus 
needed to maintain the system in its activated state (Fig. 2). Hysteresis is exploited for electromagnetic tape recording, and likewise can generate memory in biological systems. This property lends itself for converting transient biochemical responses into irreversible biological decisions, e.g. during differentiation. For example, the meiotic maturation of Xenopus oocytes requires two interrelated positive feedback loops through the ERK and the Cdc2 pathways that entertain a self-sustained activation pattern, a biological memory, from a transient stimulus. If the feedback is strong enough it can lock the system in a stable state even without further stimulation [27]. It will be interesting to examine whether such mechanisms also used for cell fate determination in mammalian systems. Engineered transcriptional units with a simply positive autoregulatory feedback loop, where the transcribed gene induces its own promoter, give rise to bistable behaviour where the transcription rate is either high or low [28]. Interestingly, while bistable behaviour was observed at the population level, single cells demonstrated a stochastic pattern of switching between the on and off states. This resembles the situation in haematopoetic differentiation [29] where single cells seem to choose stochastically between different routes of differentiation, but where still distinct populations of the correct size are generated.

Positive feedback also can destabilise systems if it becomes too strong, or generate oscillatory behaviour [8]. Typically, oscillators feature combinations of positive and negative feedback loops with time delays [30]. An example is the circadian clock where a series of coordinated feedbacks between transcriptional activation and protein degradation generate entrained oscillations. Interestingly, ERK activation is also subjected to a circadian rhythm and its oscillatory behaviour seems to be due to a negative feedback through the induction of the expression of MAPK phosphatases [31,32]. The physiological role of ERK in the 
circadian clockwork is not entirely clear yet, but it seems to link resetting of the clock in response to light input [32].

In most cases negative feedback loops tend to stabilise systems [8]. A classical example is the feedback amplifier [33], an ingeniously simple engineering device consisting of an amplifier with a negative feedback loop connecting the output with the input. For some loss of gain, fidelity and robustness increase remarkably. This has been convincingly reproduced in engineered transcriptional systems [34]. The ERK pathway resembles a feedback amplifier in many ways [11]. For instance, ERK exerts a negative feedback by interfering with Ras activation through SOS phosphorylation. SOS is an exchange factor that activates Ras by promoting the exchange of GDP for GTP. SOS forms a complex with the adaptor protein Grb2, which binds to activated tyrosine kinase receptors thereby bringing SOS into the vicinity of its substrate Ras at the cell membrane. ERK induces a phosphorylation on SOS that dissociates the Grb2-SOS complex and terminates Ras activation [35,36]. This feedback may underlie the differential activation of ERK by EGF and NGF in PC12 cells. Although it is not clear how EGF and NGF receptors would differentially activate this feedback the idea is attractive that negative feedback can stabilise a certain response pattern, in particular as the main difference between NGF and EGF on MAPK activation is a rather modest shift in the amplitude and kinetics of MAPK activation. Kinetic calculations indicate that the feedback from ERK to SOS can explain the ERK response curve to NGF treatment of PC12 cells [7].

An interesting variation on this theme was observed when analysing the differences between MAPK activation in naïve cells or cells previously and transiently exposed to a growth factor [37]. While in the naïve cells MAPK showed switch-like activation kinetics, the initiated 
cells responded with a graded MAPK activation. The reason is that a short pulse of MAPK activation induces negative feedback, in this case the expression of the MAPK phosphatase MKP-1, which is still active at the time of restimulation. Thus, the presence of a negative feedback can convert the activation kinetics of the MAPK pathway from switch-like to linear. This regulation motif is inherently attractive as it makes both the quality and the quantity of the response dependent on the history of the system, and can convert an instable into an intrinsically stable response. This also has implications for the selection of drug targets. Pathways that operate as feedback amplifiers stabilise system behaviour by smoothening out perturbations. Thus, a drug targeting a protein in the part of the pathway bracketed by a negative feedback should be less effective than a drug with a target outside of the feedback loop bracket [8]. From this point of view MEK is not a good drug target as it is in the part of the pathway that is braced by negative feedback loops, such as from ERK to MEK and ERK to SOS. This could contribute to the fact that MEK inhibitors are extremely potent on the purified enzyme in vitro, but do have much less effect in vivo [38].

\section{Scaffolds and localisation}

The important role of scaffolding proteins in the regulation of signal transduction is increasingly acknowledged [2]. By tethering pathway components together they can insulate pathways from each other, in particular when one component is used in several different pathways such as Ste11 in yeast MAPK pathways. However, they also can forge connections between pathways and distribute signals as exemplified by the large signalling complexes assembled at tyrosine kinase receptors. Scaffolds have a profound effect on the signal flux by increasing the effective local concentration of components and enhancing their interaction. While this is intuitive, mathematical modelling of scaffolding the ERK pathway has indicated the potentially enormous impact of this effect [39]. In this case, scaffolds also 
convert the double phosphorylation of ERK into a processive reaction. This should eliminate ultrasensitivity, but augments the activation rate. Of course, the contribution of these effects to the overall activation kinetics of the ERK pathway will depend on the expression level and binding affinity of the scaffold for its clients, but could explain why ultrasensitivity is not always observed in mammalian cells. On the other hand, if the concentration of the scaffold exceeds that of its client proteins, incomplete complexes will form and effectively dissipate signalling. This inhibitory effect increases with the number of clients a scaffold has. A three client scaffold such as KSR, which can bind Raf, MEK and ERK, will inhibit more effectively than a two client scaffold, such as MP-1, which binds MEK and ERK.

Another important function of scaffolds is to organise the spatial arrangement of the signalling pathway architecture. The KSR scaffolded complex forms at the cell membrane [40], whereas MP-1 targets the MEK-ERK complex to the endosomes via interaction with p14, an endosomal protein [41]. This could orchestrate the distribution of the signal by directing ERK to different substrates in different subcellular compartments. Alternatively, but not mutally exclusive such differential distribution could determine the response kinetics. Models of EGF receptor signalling that include receptor internalisation predict that endosomal signalling prevails especially at low concentrations of EGF [6,42]. Thus, the endosomal MEK-ERK complex could be a sensitivity sensor for the EGF concentration. As receptor internalisation creates a time delay the compartmentalisation of MEK-ERK complexes also could account for different activation phases with the membrane compartment being activated first and the endosomal compartment later. 


\section{Outlook}

Computational modelling can provide useful information to understand the behaviour of biological systems. It also has made a number of provocative predictions, of which only few were experimentally tested. This situation is changing as the value of the conversation between biology and the theoretical sciences is realised. A major constraint is that most of the data which biologists produce are not directly amenable to dynamic modelling. They contain sparse time series, are often qualitative rather than quantitative and show relative changes rather than changes in absolute concentrations. As there is only very little standardisation of measurements, data from different laboratories usually only can be compared in a qualitative fashion. These obstacles particularly apply to the use of ordinary differential equations (ODEs) which require absolute changes in concentrations and exact reaction rates as input. ODEs rely on this detailed information as they describe the biochemical reactions at a very fine grained level. Ideally, one would wish to move seamlessly between different levels of abstraction that can describe individual reactions, network modules and whole networks. Such methods are being currently being developed [43] and will hopefully stimulate further exploration of the wonderfully weird world of signalling networks. 


\section{References}

1. Boldt, S. and Kolch, W. (2004). Targeting MAPK signalling: Prometheus' fire or Pandora's box? Curr.Pharm.Des 10, 1885-1905.

2. Morrison, D.K. and Davis, R.J. (2003). Regulation of MAP kinase signaling modules by scaffold proteins in mammals. Annu.Rev.Cell Dev.Biol 19:91-118., 91-118.

3. Kolch, W. (2000). Meaningful relationships: the regulation of the Ras/Raf/MEK/ERK pathway by protein interactions. Biochem.J. 351, 289-305.

4. Wellbrock, C., Karasarides, M., and Marais, R. (2004). The RAF proteins take centre stage. Nat.Rev.Mol.Cell Biol 5, 875-885.

5. Vaudry, D., Stork, P.J., Lazarovici, P., and Eiden, L.E. (2002). Signaling pathways for PC12 cell differentiation: making the right connections. Science 296, 1648-1649.

6. Schoeberl, B., Eichler-Jonsson, C., Gilles, E.D., and Muller, G. (2002). Computational modeling of the dynamics of the MAP kinase cascade activated by surface and internalized EGF receptors. Nat.Biotechnol. 20, 370-375.

7. Brightman, F.A. and Fell, D.A. (2000). Differential feedback regulation of the MAPK cascade underlies the quantitative differences in EGF and NGF signalling in PC12 cells. FEBS Lett. 482, 169-174.

8. Sauro, H.M. and Kholodenko, B.N. (2004). Quantitative analysis of signaling networks. Prog.Biophys.Mol.Biol 86, 5-43.

9. Kofahl, B. and Klipp, E. (2004). Modelling the dynamics of the yeast pheromone pathway. Yeast 21, 831-850. 
10. Wiley, H.S., Shvartsman, S.Y., and Lauffenburger, D.A. (2003). Computational modeling of the EGF-receptor system: a paradigm for systems biology. Trends Cell Biol. 13, 43-50.

11. Chapman, S. and Asthagiri, A.R. (2004). Resistance to signal activation governs design features of the MAP kinase signaling module. Biotechnol.Bioeng. 85, 311-322.

12. Gong, Y. and Zhao, X. (2003). Shc-dependent pathway is redundant but dominant in MAPK cascade activation by EGF receptors: a modeling inference. FEBS Lett. \%20;554, 467-472.

13. Hatakeyama, M. et al. (2003). A computational model on the modulation of mitogenactivated protein kinase (MAPK) and Akt pathways in heregulin-induced ErbB signalling. Biochem.J. 373, 451-463.

14. Somsen, O.J., Siderius, M., Bauer, F.F., Snoep, J.L., and Westerhoff, H.V. (2002). Selectivity in overlapping MAP kinase cascades. J.Theor.Biol 218, 343-354.

15. Vayttaden, S.J., Ajay, S.M., and Bhalla, U.S. (2004). A spectrum of models of signaling pathways. Chembiochem. 5, 1365-1374.

16. Dhillon, A.S., Pollock, C., Steen, H., Shaw, P.E., Mischak, H., and Kolch, W. (2002). Cyclic AMP-Dependent Kinase Regulates Raf-1 Kinase Mainly by Phosphorylation of Serine 259. Mol.Cell Biol. 22, 3237-3246.

17. Dhillon, A.S. and Kolch, W. (2002). Untying the regulation of the Raf-1 kinase. Arch.Biochem.Biophys. 404, 3-9.

18. Eblen, S.T., Slack-Davis, J.K., Tarcsafalvi, A., Parsons, J.T., Weber, M.J., and Catling, A.D. (2004). Mitogen-activated protein kinase feedback phosphorylation 
regulates MEK1 complex formation and activation during cellular adhesion. Mol.Cell Biol 24, 2308-2317.

19. Ferrell, J.E., Jr. and Machleder, E.M. (1998). The biochemical basis of an all-or-none cell fate switch in Xenopus oocytes. Science 280, 895-898.

20. Whitehurst, A., Cobb, M.H., and White, M.A. (2004). Stimulus-coupled spatial restriction of extracellular signal-regulated kinase $1 / 2$ activity contributes to the specificity of signal-response pathways. Mol.Cell Biol 24, 10145-10150.

21. Yeung, K. et al. (1999). Suppression of Raf-1 kinase activity and MAP kinase signalling by RKIP. Nature 401, 173-177.

22. Corbit, K.C., Trakul, N., Eves, E.M., Diaz, B., Marshall, M., and Rosner, M.R. (2003). Activation of Raf-1 signaling by protein kinase C through a mechanism involving Raf kinase inhibitory protein. J.Biol Chem. 278, 13061-13068.

23. Cho, K.H., Shin, S.Y., Kim, H.W., Wolkenhauer, O., McFerran, B., and Kolch, W. (2003). Mathematical Modeling of the Influence of RKIP on the ERK Signaling Pathway. Computational Methods in Systems Biology CSMB 2003, 127-141.

24. Murphy, L.O., Smith, S., Chen, R.H., Fingar, D.C., and Blenis, J. (2002). Molecular interpretation of ERK signal duration by immediate early gene products. Nat.Cell Biol. 4, 556-564.

25. Ferrell, J.E. (2002). Self-perpetuating states in signal transduction: positive feedback, double-negative feedback and bistability. Curr.Opin.Cell Biol 14, 140-148. 
26. Kholodenko, B.N. (2000). Negative feedback and ultrasensitivity can bring about oscillations in the mitogen-activated protein kinase cascades. Eur.J Biochem. 267, 1583-1588.

27. Xiong, W. and Ferrell, J.E., Jr. (2003). A positive-feedback-based bistable 'memory module' that governs a cell fate decision. Nature 426, 460-465.

28. Becskei, A., Seraphin, B., and Serrano, L. (2001). Positive feedback in eukaryotic gene networks: cell differentiation by graded to binary response conversion. EMBOJ. $20,2528-2535$.

29. Hoang, T. (2004). The origin of hematopoietic cell type diversity. Oncogene $\% 20 ; 23$, 7188-7198.

30. Goldbeter, A. (2002). Computational approaches to cellular rhythms. Nature 420, $238-245$.

31. Hayashi, Y., Sanada, K., and Fukada, Y. (2001). Circadian and photic regulation of MAP kinase by Ras- and protein phosphatase-dependent pathways in the chick pineal gland. FEBS Lett. 491, 71-75.

32. Butcher, G.Q., Lee, B., and Obrietan, K. (2003). Temporal regulation of lightinduced extracellular signal-regulated kinase activation in the suprachiasmatic nucleus. J.Neurophysiol. 90, 3854-3863.

33. Black, H.S. (1977). Inventing the negative feedback amplifier. IEEE Spectrum 14, $55-60$.

34. Becskei, A. and Serrano, L. (2000). Engineering stability in gene networks by autoregulation. Nature 405, 590-593. 
35. Douville, E. and Downward, J. (1997). EGF induced SOS phosphorylation in PC12 cells involves P90 RSK-2. Oncogene 15, 373-383.

36. Langlois, W.J., Sasaoka, T., Saltiel, A.R., and Olefsky, J.M. (1995). Negative feedback regulation and desensitization of insulin- and epidermal growth factorstimulated p21ras activation. J.Biol Chem. 270, 25320-25323.

37. Bhalla, U.S., Ram, P.T., and Iyengar, R. (2002). MAP kinase phosphatase as a locus of flexibility in a mitogen-activated protein kinase signaling network. Science 297, 1018-1023.

38. Rinehart, J. et al. (2004). Multicenter phase II study of the oral MEK inhibitor, CI1040, in patients with advanced non-small-cell lung, breast, colon, and pancreatic cancer. J.Clin.Oncol. 22, 4456-4462.

39. Levchenko, A., Bruck, J., and Sternberg, P.W. (2000). Scaffold proteins may biphasically affect the levels of mitogen-activated protein kinase signaling and reduce its threshold properties. Proc.Natl.Acad.Sci.U.S.A 97, 5818-5823.

40. Muller, J., Ory, S., Copeland, T., Piwnica-Worms, H., and Morrison, D.K. (2001). CTAK1 regulates Ras signaling by phosphorylating the MAPK scaffold, KSR1. Mol.Cell 8, 983-993.

41. Teis, D., Wunderlich, W., and Huber, L.A. (2002). Localization of the MP1-MAPK scaffold complex to endosomes is mediated by p14 and required for signal transduction. Dev.Cell 3, 803-814. 
42. Resat, H., Ewald, J.A., Dixon, D.A., and Wiley, H.S. (2003). An integrated model of epidermal growth factor receptor trafficking and signal transduction. Biophys.J. 85, 730-743.

43. Calder, M., Hillston, J., and Gilmore, S. (2004). Modelling the influence of RKIP on the ERK signalling pathway using the stochastic process algebra PEPA. Proceedings of Bio-CONCUR 2004, Electronic Notes in Theoretical Computer Science

\section{Figure legends}

Figure 1. The MAPK pathway and feedback regulation. Ras and proteins involved in Ras activation are displayed in shades of blue, kinases in shades of red, inhibitory proteins in shades of grey and transcription factors in turquoise. Green arrows represent activation, red lines with blunt ends represent inhibition. See text for details.

Figure 2. Non-linear relationships between stimulation and activation. Schematic representation of input / output curves characteristic for enzymes displaying MichaelisMenten kinetics, ultrasensitivity, hysteresis and bistability. See text for details. 
Figure 1

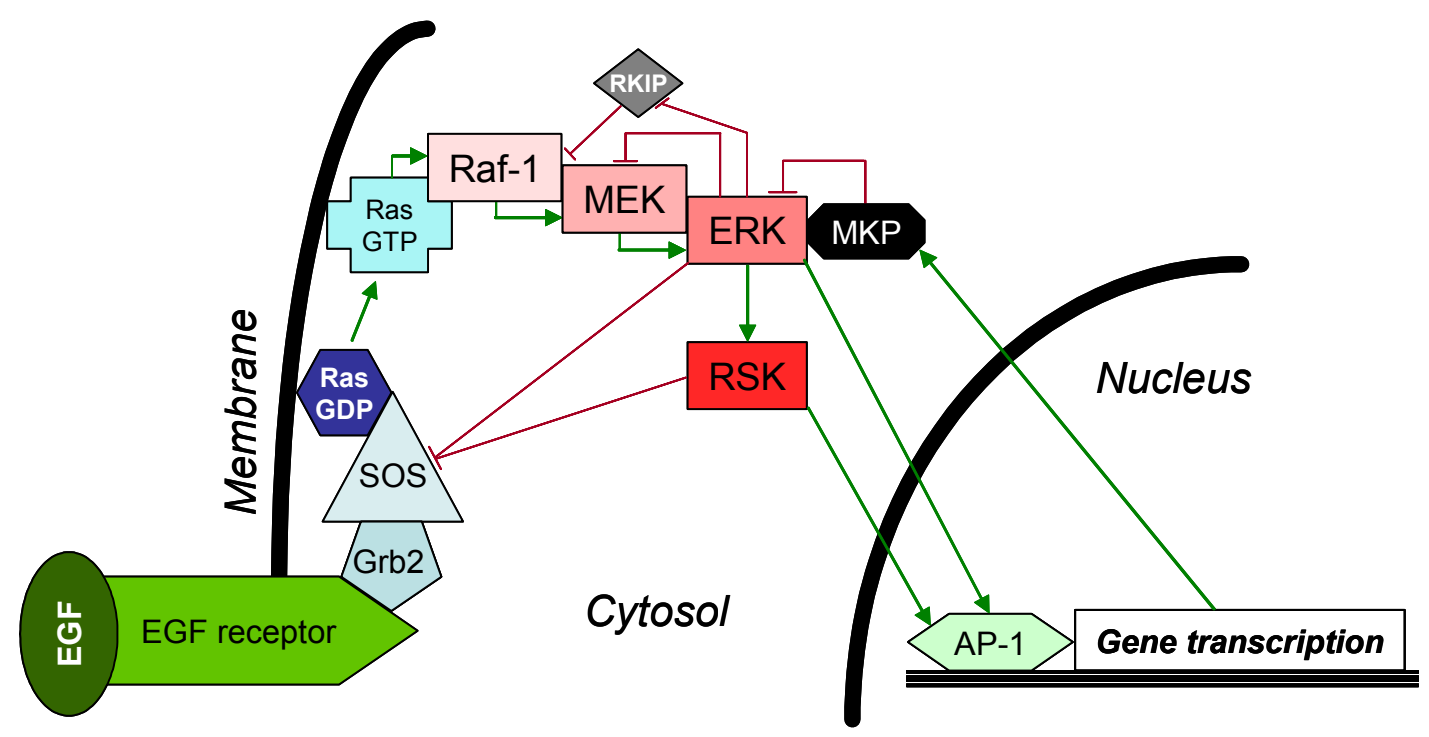

Figure 2

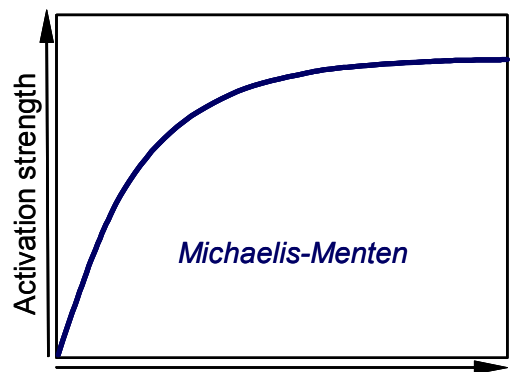

Stimulation strength

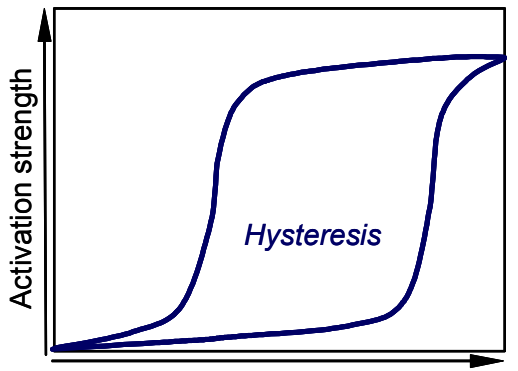

Stimulation strength

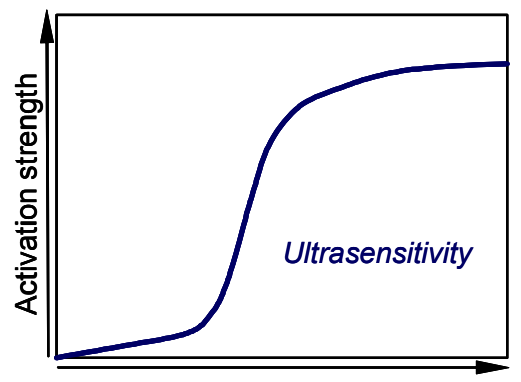

Stimulation strength

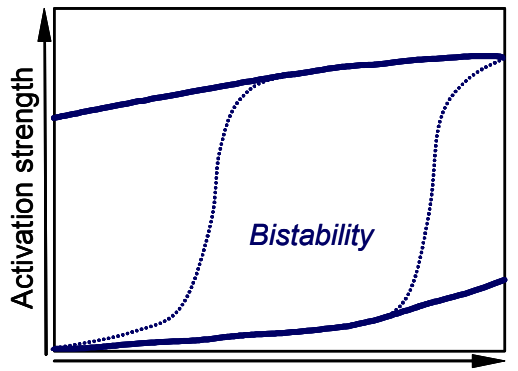

Stimulation strength 